\title{
DEFORMATIONS OF OPEN STEIN MANIFOLDS
}

\author{
CHARLES L. EPSTEIN AND YONG OUYANG
}

\begin{abstract}
We consider the deformation of the complex structure on an open Stein manifold. We show that a tame, compactly supported deformation of a Stein manifold is trivial. The remainder of our results are for deformations of the standard complex structure on $\mathbb{C}^{n}$. A deformation of $\mathbb{C}^{n}$ which tends to a constant deformation faster that $r^{-3}$ is trivial. Harmonic deformation tensors (w.r.t to the standard Euclidean metric) which are regular at infinity are constant.
\end{abstract}

\section{INTRODUCTION}

The deformation theory of the complex structure on higher dimensional compact manifolds is very well understood, principally from the work of Kodaira and Spencer and Kuranishi, see [9] and [10]. The deformation theory of Riemann surfaces, both open and compact is also very well understood, see for example [2]. The pioneering work of Ahlfors and Bers places the 'Beltrami coefficient' at the center of the discussion. If $z$ is a local holomorphic parameter then a Beltrami coefficient is given locally as $\mu \partial_{z} \otimes d \bar{z}$, where $\mu$ is a function. It is evidently a deformation tensor for the complex structure. A function $w(z, \bar{z})$ is holomorphic with respect to the deformed complex structure if and only if

$$
\partial_{\bar{z}} w+\mu \partial_{z} w=0 .
$$

Because there is no integrability condition for complex structures in one dimension it is possible to work with deformation tensors which are merely measureable. This is quite a useful fact as it often allows one to reduce to compactly supported deformations: one simply replaces $\mu$ by $\chi_{B_{r}} \mu$ and then allows $r \rightarrow \infty$. In higher dimensions there is, at present no effective way to reduce to compactly supported deformations. The basic tool for studying deformation problems in one dimension is the measurable Riemann mapping theorem, see [1]. If $\mu$ is a measurable Beltrami coefficient defined on $\mathbb{C}$ such that $\|\mu\|_{L^{\infty}}<k<1$, then this result implies that there is a homeomorphism $w: \mathbb{C} \rightarrow \mathbb{C}$ satisfying (1) 
on all of $\mathbb{C}$. In other words $\mathbb{C}$ with the deformed complex structure is biholomorphic to the standard complex plane.

The deformation theory of open Stein manifolds in higher dimensions is much less developed. Hamilton showed that if $Y$ is a relatively compact domain, with smooth boundary in a complex manifold $X$, such that $H^{1}(Y ; \Theta)=0(\Theta$ is the tangent sheaf $)$ then all sufficiently small deformations of the complex structure on $Y$, smooth up to the boundary, are obtained from smooth deformations of the embedding of $Y$ into $X$, see [4] Colloquially one says that all sufficiently small deformations of the complex structure on $Y$ are 'wiggles.' Kiremidjian extended Kuranishi's results to a class of manifolds which are, in essence bounded domains in Kähler manifolds, see [8].

In this note we prove several results about deformation theory of open Stein manifolds, in particular $\mathbb{C}^{n}$. Our first result concerns deformations of Stein manifolds with compact support: If $\Omega$ is an integrable deformation tensor on a Stein manifold with (1) compact support and (2) norm less than 1, in an appropriate sense, then the complex structure it defines is equivalent to the undeformed structure. The remainder of our results concern deformations of the complex structure on $\mathbb{C}^{n}, \quad n \geq 2$. We next show that an integrable deformation of the standard complex structure on $\mathbb{C}^{n}$ which decays at infinity faster than $|z|^{-3}$ is equivalent to the standard structure.

In the context of the deformation theory of compact manifolds, Kuranishi introduced the notion of an harmonic deformation tensor. These are deformation tensors which satisfy the standard integrability condition (5) and also the additional equation $\bar{\partial}^{*} \Omega=0$. In the context of compact manifolds the space of solutions to this system of equations is actually the base space for a versal deformation, see [10]. We show that a harmonic deformation tensor on $\mathbb{C}^{n}$ which approaches a constant rapidly enough is actually constant. We also show that harmonic deformation tensors with sufficiently small norm which are regular at infinity, in the sense that they have a smooth extension to the radial compactification of $\mathbb{C}^{n}$ are also constant.

The results in this note are adaptations of those in the thesis of the second author. More results of this type can be found there, see [12].

\section{Notation AND TERMinology}

In this section we fix the notation and terminology we use thoughout the paper. These results are all well known and can be found, for example in [8]. The complex structure on a smooth manifold, $X$ can be described as subbundle, $T^{1,0} X$ of $T X \otimes \mathbb{C}$. Let $T^{0,1} X$ denote the 
conjugate subbundle. Such a subbundle defines an almost complex structure if it satisfies the following conditions

(a) $\quad T^{1,0} X \oplus T^{0,1} X=T X \otimes \mathbb{C}$.

(b) $T^{1,0} X \cap T^{0,1} X$ is the zero section of $T X \otimes \mathbb{C}$,

It defines a complex structure if it also satisfies the following integrability condition:

(c) If $Z, W \in \mathcal{C}^{\infty}\left(X ; T^{1,0} X\right)$ then $[Z, W] \in \mathcal{C}^{\infty}\left(X ; T^{1,0} X\right)$ as well.

A choice of almost complex structure determines an orientation for $X$ as follows: For $x \in X$ let $\left\{Z_{1}, \ldots, Z_{n}\right\}$ be a frame for $T_{x}^{1,0} X$; then $\left\{\Re Z_{1}, \Im Z_{1}, \ldots, \Re Z_{n}, \Im Z_{n}\right\}$ defines an oriented frame for $T_{x} X$. A simple calculation shows that a different choice of frame for $T_{x}^{1,0} X$ leads to a frame for $T_{x} X$ in the same orientation class. We call this the orientation induced by the choice of almost complex structure.

When discussing deformations of complex structures it is customary to work with the conjugate bundle $T^{0,1} X$. We now suppose that a subbundle $T^{0,1} X$ defining a complex structure is fixed. We call this the reference complex structure. Let $\pi_{1,0}, \pi_{0,1}$ be the projections onto the indicated subbundles. Let ${ }^{\prime} T^{0,1} X$ be another subbundle of $T X \otimes \mathbb{C}$ which satisfies conditions a. and b. above.

Definition 1. If ' $T^{1,0} X$ induces the same orientation of $X$ as $T^{1,0} X$ and the restriction

$$
{ }^{\prime} \pi_{0,1}:{ }^{\prime} T^{0,1} X \longrightarrow T^{0,1} X
$$

is an isomorphism then we say that ${ }^{\prime} T^{0,1} X$ is a finite distance from $T^{0,1} X$.

Note that the orientation preserving diffeomorphisms of $X, \operatorname{Diff}^{+}(X)$ acts on the set of almost complex structures: If $\varphi \in \operatorname{Diff}^{+}(X)$ and ${ }^{\prime} T^{0,1} X$ is an almost complex structure then we define a new almost complex structure on $X$ by setting

$$
{ }^{\prime \prime} T_{\varphi(x)}^{0,1} X=\varphi_{*}^{\prime} T_{x}^{0,1} X
$$

for each $x \in X$. If ' $T^{0,1} X$ is integrable then so is its image under $\varphi$. Even if ' $T^{0,1} X$ is a finite distance from $T^{0,1} X$, its image under $\varphi$ may not be. For example: if $X=\mathbb{C}^{2}$ and $C(z, w)=(\bar{z}, \bar{w})$ then $C_{*} T^{0,1} \mathbb{C}^{2}=T^{1,0} \mathbb{C}^{2}$, which is clearly not a finite distance from $T^{0,1} \mathbb{C}^{2}$. On the other hand, the complex conjugation map can be joined to the identity map by a smooth family of linear diffeomorphisms of $\mathbb{R}^{4}$.

Definition 2. Two almost complex structures which are related by a diffeomorphism as in (3) are called equivalent. A deformation which is equivalent to the reference structure is called trivial. 
Note that if two complex structures are equivalent then the diffeomorphism, $\varphi$ in (3) is a biholomorphic mapping:

$$
\varphi:\left(X,{ }^{\prime} T^{0,1} X\right) \longrightarrow\left(X,{ }^{\prime \prime} T^{0,1} X\right) .
$$

Definition 3. If ' $\pi_{0,1}$ is an isomorphism then we define the deformation tensor $\Omega \in \mathcal{C}^{\infty}\left(X ; \operatorname{hom}\left(T^{0,1} X, T^{1,0} X\right)\right)$ by

$$
\Omega(\bar{Z})=\pi_{1,0} \circ^{\prime} \pi_{0,1}^{-1}(\bar{Z}) .
$$

In this case

$$
{ }^{\prime} T_{x}^{0,1} X=\left\{\bar{Z}+\Omega(\bar{Z}): \bar{Z} \in T_{x}^{0,1} X\right\}
$$

and we denote ${ }^{\prime} T^{0,1} X$ by ${ }^{\Omega} T^{0,1} X$.

If $\operatorname{dim} X=1$ then $\Omega$ is just a Beltrami coefficient. If $\Omega$ is a deformation tensor and $\varphi \in \operatorname{Diff}^{+}(X)$ is such that $\varphi_{*}{ }^{\Omega} T^{0,1} X$ is also at finite distance from the reference structure then we denote the deformation tensor for this structure by $\varphi_{\#}(\Omega)$.

We need to rephrase conditions (b) and (c) in terms of $\Omega$. Condition (b) becomes

(b') The nullspace of $\mathrm{Id}-\bar{\Omega} \circ \Omega$ is trivial.

Condition (c) becomes

(c') $\Omega$ satisfies the integrability equation

$$
\bar{\partial} \Omega-\frac{1}{2}[\Omega, \Omega]=0 .
$$

If $\left\{z_{1}, \ldots, z_{n}\right\}$ are local holomorphic coordinates so that

$$
\Omega=\sum_{i, j=1}^{n} a_{i j} \partial_{z_{i}} \otimes d \bar{z}_{j}
$$

then

$$
[\Omega, \Omega]=\sum_{i, j, k, l}\left[a_{i j} \partial_{z_{i}}\left(a_{k l}\right) \partial_{z_{k}}+a_{k l} \partial_{z_{k}}\left(a_{i j}\right) \partial_{z_{i}}\right] \otimes d \bar{z}_{j} \wedge d \bar{z}_{l}
$$

If $A \in \mathrm{Gl}_{n}(\mathbb{C})$ then an elementary computation shows that we have the identities:

$$
\operatorname{Tr}(A \bar{A})^{k}=\operatorname{Tr}(\bar{A} A)^{k}=\overline{\operatorname{Tr}(A \bar{A})^{k}} \text { for } k \in \mathbb{N} .
$$

Using the relationship between the trace powers and the elementary symmetric functions this implies that $\operatorname{det}(\operatorname{Id}-\bar{A} \circ A)=0$ is a real equation, let $\mathcal{Z}$ denote the zero locus. It contains hypersurface components; we let $\mathcal{C}_{0}$ denote the component of the complement of $\mathcal{Z}$ which contains $A=0$. If we interpret $A$ as a map from $T^{0,1} \mathbb{C}^{n}$ to $T^{1,0} \mathbb{C}^{n}$ then $\mathcal{Z}$ is invariant under changes of basis and therefore it makes sense to say that $\Omega_{x} \in \mathcal{C}_{0}$ for an $x \in X$. In order for the deformed complex 
structure ${ }^{\Omega} T^{0,1} X$ to induce the same orientation as the reference structure it is sufficient that $\Omega_{x}$ belong to $\mathcal{C}_{0}$ for all $x \in X$. If $\operatorname{dim}_{\mathbb{C}} X=1$ then $\bar{\Omega} \circ \Omega$ is a scalar and this condition is simply that

$$
\|\Omega(x)\|=\sqrt{\bar{\Omega}_{x} \circ \Omega_{x}}<1 .
$$

In higher dimensions $\bar{\Omega} \circ \Omega$ is not a scalar thus we need to select a metric in order to measure its size. Let $g$ be a hermitian metric on $X$ and define $\left|\Omega_{x}\right|_{g}$ as the operator norm of the map $\Omega_{x}: T_{x}^{0,1} X \rightarrow T_{x}^{1,0} X$ :

$$
\left|\Omega_{x}\right|_{g}=\max _{T_{x}^{0,1} X \ni \bar{Z} \neq 0} \frac{\left|\Omega_{x}(\bar{Z})\right|_{g}}{|\bar{Z}|_{g}}
$$

and

$$
\|\Omega\|_{g}=\sup _{x \in X}\left|\Omega_{x}\right|_{g}
$$

Definition 4. A deformation tensor $\Omega$ is tame (w.r.t $g$ ) if $\|\Omega\|_{g}<1$.

This easily implies that ${ }^{\Omega} T^{0,1} X$ is a finite distance from $T^{0,1} X$.

\section{Compactly supported Deformations}

Let $X$ be a Stein manifold and let $\psi$ denote a smooth, strictly plurisubharmonic, exhaustion function defined on $X$. The $(1,1)$-form

$$
\omega=i \partial \bar{\partial} \psi=d d^{c} \psi
$$

defines a Kähler form. Let $g$ denote the associated hermitian metric on the tangent space, it is not necessary for $\psi$ to tend to infinity or for $g$ to be complete.

Theorem 1. Let $\Omega \in \mathcal{C}^{\infty}\left(X ; \operatorname{hom}\left(T^{0,1} X, T^{1,0} X\right)\right)$ be a compactly supported, integrable deformation tensor such that $\Omega$ is tame w.r.t. $g$ then there exists a $\varphi \in \operatorname{Diff}_{c}^{+}(X)$ such that

$$
\varphi_{\#}(\Omega)=0 \text {. }
$$

That is: $\left(X,{ }^{\Omega} T^{0,1} X\right)$ is equivalent to $\left(X, T^{0,1} X\right)$.

Proof. Let $K$ denote the support of $\Omega$. Since $K$ is compact there is real number $c$, not a critical value of $\psi$ such that $K \subset \psi^{-1}((-\infty, c))=D$. Let $M=b D=\psi^{-1}(c)$. This is a smooth, connected manifold as $D$ is a relatively compact subet of $X$ with a strictly pseudoconvex boundary. To avoid confusion we let $D^{\prime}$ denote $D$ with the complex structure ${ }^{\Omega} T^{0,1} X$. As the deformation tensor vanishes in a neighborhood, $U$ of $b D$ the identity map from $U$ to $U$ is biholomorphic (from ${ }^{\Omega} T^{0,1}$ to $T^{0,1}$ ). Since $D^{\prime}$ is a holomorphically convex, normal, complex space and $D$ is Stein Id $\left.\right|_{U}$ has an holomorphic extension as a map $\varphi: D^{\prime} \rightarrow D$. To 
show that this extension is actually a biholomorphism it suffices to show that $D^{\prime}$ is itself Stein. As $D^{\prime}$ has a strictly pseudoconvex boundary, Grauert's theorem, see [3] shows that this is equivalent to showing that the maximal compact analytic subset of $D^{\prime}$ is zero dimensional.

To that end we prove a lemma.

Lemma 1. If $\Omega$ satisfies the hypotheses of the theorem and $L \subset T_{x} X$ is a $2 k$-dimensional subspace which is complex with respect to the deformed complex structure then, with the orientation induced by ${ }^{\Omega} T^{1,0} X$, we have that

$$
\left.\omega^{k}\right|_{L}>0
$$

Before proving the lemma we show how it completes the proof of the theorem. Suppose that $D^{\prime}$ has a positive dimensional, compact analytic subset. This implies that for some $k>0, D^{\prime}$ contains a pure $k$-dimensional, compact, ${ }^{\Omega} T^{0,1}$-analytic subset $Y$. Using the orientation induced by the deformed complex structure, $Y$ defines $2 k$-dimensional, closed current of integration $[Y]$, see [5]. From the lemma it follows immediately that

$$
\left\langle\omega^{k},[Y]\right\rangle>0
$$

On the other hand if $\alpha=d^{c} \psi$ then $\omega^{k}=d\left(\alpha \wedge \omega^{k-1}\right)$ and therefore as $[b Y]=0$ we have that

$$
\left\langle\omega^{k},[Y]\right\rangle=\left\langle d\left(\alpha \wedge \omega^{k-1}\right),[Y]\right\rangle=\left\langle\alpha \wedge \omega^{k-1},[b Y]\right\rangle=0 .
$$

This contradiction proves that $\varphi: D^{\prime} \rightarrow D$ is a biholomorphism. As $\varphi$ reduces to the identity in a neighborhood of $b D$ we can extend it to $X \backslash D$ as the identity and obtain a compactly supported diffeomorphism satisfying the conclusion of the theorem.

Proof of the lemma. Let $\xi$ denote $\left.\omega\right|_{L}$. Because $\omega$ is a real form, $\xi$ can be decomposed into $(p, q)$-types with respect to the deformed complex structure as

$$
\xi=\xi_{1}+\xi_{2}+\bar{\xi}_{2}
$$

Here $\xi_{1}$ is a $(1,1)$-covector and $\xi_{2}$ is a $(2,0)$-covector. Let $\bar{Z}^{\prime}=\bar{Z}+\Omega(\bar{Z})$ belong to $L$ then

$$
\begin{aligned}
\xi_{1}\left(Z^{\prime}, \bar{Z}^{\prime}\right) & =\omega\left(Z^{\prime}, \bar{Z}^{\prime}\right) \\
& =|\bar{Z}|_{g}^{2}-|\Omega(\bar{Z})|_{g}^{2} \\
& \geq|\bar{Z}|_{g}^{2}\left(1-\|\Omega\|_{g}^{2}\right)>0
\end{aligned}
$$

The last inequality follows as $|\bar{Z}|_{g}>0$ and $\|\Omega\|_{g}<1$. Thus $\xi_{1}$ defines a positive definite Hermitian form on $L^{1,0}$. Using simultaneous diagonalization we can find a basis $\left\{\bar{Z}_{i}^{\prime}=\bar{Z}_{i}+\Omega\left(\bar{Z}_{i}\right), i \leq k\right\}$ for $L^{0,1}$ such 
that

$$
g\left(Z_{i}^{\prime}, \bar{Z}_{j}^{\prime}\right)=\delta_{i j} \text { and } \xi_{1}\left(Z_{i}^{\prime}, \bar{Z}_{j}^{\prime}\right)=t_{i} \delta_{i j}
$$

with $t_{i}>0$. Let $\left\{Y_{i}, i \leq k\right\}$ be the dual basis to $\left\{Z_{i}^{\prime}, i \leq k\right\}$. Then $\xi_{1}$, and $\xi_{2}$ can be written as

$$
\begin{aligned}
\xi_{1} & =\sum_{i=1}^{k} \sqrt{-1} t_{i} Y_{i} \wedge \bar{Y}_{i}, \\
\xi_{2} & =\sum_{i<j} C_{i j} Y_{i} \wedge Y_{j} .
\end{aligned}
$$

The induced orientation on $L$ is given by

$$
\chi=(\sqrt{-1})^{k} Y_{1} \wedge \bar{Y}_{1} \wedge \cdots \wedge Y_{k} \wedge \bar{Y}_{k}
$$

We show that $\xi^{k}$ is positive with respect to this orientation. We have that

$$
\begin{aligned}
\xi^{k} & =\left(\xi_{1}+\xi_{2}+\bar{\xi}_{2}\right)^{k} \\
& =\sum_{p+q+r=k} \frac{k !}{p ! q ! r !} \xi_{1}^{p} \wedge \xi_{2}^{q} \wedge \bar{\xi}_{2}^{r}
\end{aligned}
$$

Since $L$ is complex with respect to the deformed structure any term with $q \neq r$ vanishes. In terms of the dual coframe we have that

$$
\xi_{1}^{p}=(\sqrt{-1})^{p} p ! \sum_{1 \leq j_{1}<\cdots<j_{p} \leq k} t_{j_{1}} \ldots t_{j_{p}} Y_{j_{1}} \wedge \bar{Y}_{j_{1}} \wedge \cdots \wedge \bar{Y}_{j_{p}}
$$

and

$$
\xi_{2}^{q}=\sum_{1 \leq i_{1}<\cdots<i_{2 q} \leq k} C\left(q, i_{1}, \ldots, i_{2 q}\right) Y_{i_{1}} \wedge \cdots \wedge Y_{i_{2 q}}
$$


These formulæ and the observation above imply that

$$
\begin{aligned}
\xi^{k}= & \left(\xi_{1}+\xi_{2}+\bar{\xi}_{2}\right)^{k} \\
= & \sum_{p+2 q=k} \frac{k !}{p ! q ! q !} \xi_{1}^{p} \wedge \xi_{2}^{q} \wedge \bar{\xi}_{2}^{q} \\
= & \sum_{p+2 q=k} \frac{k !}{q ! q !} \sum_{j_{1}<\cdots<j_{p}} \sum_{i_{1}<\cdots<i_{2 q}}\left|C\left(q, i_{1}, \ldots, i_{2 q}\right)\right|^{2} t_{j_{1}} \ldots t_{j_{p}}(\sqrt{-1})^{p} \\
& Y_{j_{1}} \wedge \bar{Y}_{j_{1}} \wedge \cdots \wedge Y_{j_{p}} \wedge \bar{Y}_{j_{p}} \wedge Y_{i_{1}} \wedge \cdots \wedge Y_{i_{2 q}} \wedge \bar{Y}_{i_{1}} \wedge \cdots \wedge \bar{Y}_{i_{2 q}} \\
= & \sum_{p+2 q=k} \frac{k !}{q ! q !} \sum_{j_{1}<\cdots<j_{p}} \sum_{i_{1}<\cdots<i_{2 q}}\left|C\left(q, i_{1}, \ldots, i_{2 q}\right)\right|^{2} t_{j_{1}} \ldots t_{j_{p}}(\sqrt{-1})^{p+2 q} \\
& Y_{j_{1}} \wedge \bar{Y}_{j_{1}} \wedge \cdots \wedge Y_{j_{p}} \wedge \bar{Y}_{j_{p}} \wedge Y_{i_{1}} \wedge \bar{Y}_{i_{1}} \wedge \cdots \wedge Y_{i_{2 q}} \wedge \bar{Y}_{i_{2 q}} \\
\geq & k ! t_{1} \ldots t_{k}(\sqrt{-1})^{k} Y_{1} \wedge \bar{Y}_{1} \wedge \ldots Y_{k} \wedge \bar{Y}_{k} .
\end{aligned}
$$

The estimate follows as each term

$$
(\sqrt{-1})^{k} Y_{j_{1}} \wedge \bar{Y}_{j_{1}} \wedge \cdots \wedge Y_{i_{2 q}} \wedge \bar{Y}_{i_{2 q}}
$$

is either 0 or $\chi$ and $t_{i}>0$ for $1 \leq i \leq k$. Thus we have proved that $\omega^{k}$ is positive on $L$.

Remark 1. If we fix a strictly pseudoconvex subset $Y \subset \subset X$ then Hamilton's theorem implies that any sufficiently small deformation of the complex structure of $X$ with support in $Y$ is trivial.

\section{Deformations of $\mathbb{C}^{n}$ Which DECAY AT INFINITY}

We now consider deformations of the standard complex structure on $\mathbb{C}^{n}$. In [11] Mok, Siu and Yau give the following characterization of $\mathbb{C}^{n}$ :

Theorem 2. Let $M$ be a complete Kähler manifold of complex dimension $n$ with a pole. Let $r(q)$ denote the geodesic distance from the pole $p$. Suppose $n \geq 2$ and the sectional curvatures of $M$ are bounded by

$$
-\frac{A_{\epsilon}}{(1+r)^{2+\epsilon}} \leq K \leq \frac{A_{\epsilon}}{(1+r)^{2+\epsilon}}
$$

where $A_{\epsilon}$ is a sufficiently small constant depending on $\epsilon$. Then $M$ is biholomorphic to $\mathbb{C}^{n}$.

In our next result we translate the hypotheses of this theorem into hypotheses on a deformation tensor for $\mathbb{C}^{n}$. In this section $e$ denotes the standard Euclidean inner product on $\mathbb{C}^{n}$. 
Theorem 3. Suppose that $\Omega=\sum a_{i j} \partial z_{i} \otimes d \bar{z}_{j}$ defines an integrable deformation of the standard complex structure on $\mathbb{C}^{n}$. Given $\epsilon>0$ there exists $a k$ and $\delta$ such that if

$$
\|\Omega\|_{e} \leq k<1
$$

and

$$
\left|D^{\alpha} a_{i j}(z)\right| \leq \frac{\delta}{(1+|z|)^{3+\epsilon}} \quad i, j=1, \ldots, n
$$

for all $\alpha$ such that $1 \leq|\alpha| \leq 3$

then $\left(\mathbb{C}^{n},{ }^{\Omega} T^{1,0} \mathbb{C}^{n}\right)$ is biholomorphic to $\mathbb{C}^{n}$ with the standard complex structure.

Proof. Let

$$
\bar{L}_{i}=\frac{\partial}{\partial \bar{z}_{i}}+\sum_{j} a_{i j} \frac{\partial}{\partial z_{j}}, \quad i=1, \ldots n
$$

and $\left\{\eta_{j}: j=, 1 \ldots, n\right\}$ be the dual basis of $(0,1)$-forms. With

$$
u=\sum_{i=1}^{n}\left|z_{i}\right|^{2}
$$

we have

$$
\partial_{\Omega} \bar{\partial}_{\Omega} u=\sum h_{i j} \eta^{i} \wedge \bar{\eta}^{j},
$$

where $\left(h_{i j}\right)$ is an Hermitian matrix. If $k$ and $\delta$ are sufficiently small then $h_{i j}$ is positive definite and

$$
\widetilde{\omega}=\sum h_{i j} \omega^{i} \bar{\omega}^{j}=g+i S .
$$

is a Kähler metric on $\mathbb{C}^{n}$ with respect to the deformed complex structure. Here $g$ and $S$ are real bilinear forms with

$$
g=\sum_{i, j=1}^{2 n} g_{i j} d x_{i} d x_{j}
$$

the Riemannian metric on $\mathbb{C}^{n}$ defined by the Kähler form $\widetilde{\omega}$. The hypotheses of the theorem imply the estimates

$$
\left|D^{\alpha}\left(g_{i j}(z)-g_{i j}(0)\right)\right| \leq \frac{C(\delta)}{(1+|z|)^{2+\epsilon}}, \text { for } 1 \leq|\alpha| \leq 2 .
$$

Which easily imply that $g$ is a complete metric, quasi-isometric (by the identity map) to the Euclidean metric on $\mathbb{C}^{n}$. If $r(z)$ denotes the distance from $z$ to the origin with respect to $g$ then

$$
c_{1}|z| \leq r(z) \leq c_{2}|z|
$$


where $c_{1}>0$ and $c_{2}>0$ are constants depending only on $k, \epsilon$ and $n$.

Proposition 1. Under the hypotheses of Theorem 3, the metric g defined in (10) is a complete Kähler metric with a pole at $O$. If $r$ denotes the geodesic distance from $O$ with respect to $g$ then the sectional curvatures of $g$ satisfy

$$
-\frac{C(\delta)}{(1+r)^{2+\epsilon}} \leq K \leq \frac{C(\delta)}{(1+r)^{2+\epsilon}}
$$

$C(\delta)$ depends only on $n$ and $\delta$ and $\lim _{\delta \rightarrow 0} C(\delta)=0$.

To prove this proposition, we use the following elementary lemma whose proof is left to the reader.

Lemma 2. Suppose that

$$
\partial_{t}^{2} u(t)=-\frac{a u(t)}{(1+t)^{2}}, \quad u(0)=0, \partial_{t} u(0)=1
$$

where $a<\frac{1}{4}$ is a constant, then $u(t)>0$ for all $t>0$.

To use the lemma we employ the following version of the Sturm comparison theorem.

Lemma 3. For $0 \leq t<\infty$ suppose that

$$
\begin{aligned}
& \partial_{t}^{2} f(t)+K(t) f(t) \geq 0 \\
& \partial_{t}^{2} \widetilde{f}(t)+\tilde{K}(t) \tilde{f}(t)=0 \text { and } \\
& \quad f(0)=\tilde{f}(0)=0, \quad \partial_{t} f(0)=\partial_{t} \tilde{f}(0)=1 .
\end{aligned}
$$

If $\tilde{K(} t) \geq K(t)$ for all $0 \leq t<\infty$ and $\tilde{f}(t)>0$ for $t>0$, then $f(t) \geq \tilde{f}(t)$ for all $t$.

Proof of the proposition. Step 1. We first estimate the sectional curvature. If $\Gamma_{i j}^{k}$ denote the Christoffel symbols of the metric $g$ then it follows from (11) that we have the estimates

$$
\left|D^{\alpha} \Gamma_{i j}^{k}\right| \leq \frac{C(\delta)}{(1+|z|)^{2+\epsilon}} \text { for }|\alpha| \leq 1 .
$$

The Riemann curvature tensor therefore satisfies

$$
\left|R_{i j k}^{s}\right| \leq \frac{C(\delta)}{(1+|z|)^{2+\epsilon}},
$$

It again follows from (11) that

$$
\left|R_{i j k l}\right|=\left|\sum_{s} R_{i j k}^{s} g_{s l}\right| \leq \frac{C(\delta)}{(1+|z|)^{2+\epsilon}} \leq \frac{C(\delta)}{(1+r(z))^{2+\epsilon}}
$$


which proves the estimates (13).

Step 2. We now show that $O$ is a pole. Let $\gamma(t)$ be an arclength parametrized, geodesic emanating from the origin $O$ and $J$ a Jacobi field along $\gamma$ which satisfies $J(0)=0, D_{t} J(0)=v \neq 0$. The Jacobi field satisfies the following second order ODE

$$
\frac{D^{2} J(t)}{d t^{2}}+R\left(\gamma^{\prime}(t), J(t)\right) \gamma^{\prime}(t)=0 \text {. }
$$

Let $\left\{e_{1}(t), \ldots, e_{2 n}(t)\right\}$ be parallel, orthonormal fields along $\gamma(t)$. With

$$
\begin{gathered}
J(t)=\sum_{i} f_{i}(t) e_{i}(t), \\
a_{i j}=<R\left(\gamma^{\prime}(t), e_{i}(t)\right) \gamma^{\prime}(t), e_{j}(t)>,
\end{gathered}
$$

we rewrite the Jacobi equation as

$$
\partial_{t}^{2} f_{j}(t)+\sum_{i} a_{i j}(t) f_{i}(t)=0, j=1, \ldots, 2 n .
$$

It follows from (13) that $a_{i j}(t)$ satisfies the estimates

$$
\left|a_{i j}(t)\right| \leq \frac{C(\delta)}{(1+t)^{2+\epsilon}}
$$

With $\phi(t)=\left(\sum_{j} f_{j}^{2}(t)\right)^{\frac{1}{2}}$ we obtain that

$$
\partial_{t}^{2} \phi(t)=\frac{\sum_{i}\left(\left(\partial_{t} f_{i}\right)^{2}+f_{i} \partial_{t}^{2} f_{i}\right)}{\phi}-\frac{\left(\sum_{i} f_{i} \partial_{t} f_{i}\right)^{2}}{\phi^{3}}
$$

Using the Cauchy-Schwarz inequality on the second sum we obtain that

$$
\begin{aligned}
\partial_{t}^{2} \phi(t) & \geq \frac{\sum_{i}\left(\partial_{t} f_{i}\right)^{2}+\sum_{i, j} a_{i j} f_{i} f_{j}}{\phi}-\frac{\phi^{2} \sum_{i}\left(\partial_{t} f_{i}\right)^{2}}{\phi^{3}} \\
& \geq-\frac{C(\delta)}{(1+t)^{2+\epsilon}} \phi(t) .
\end{aligned}
$$

For small $t$ we have that $f(t)=t v+O\left(t^{2}\right)$ and therefore $\phi(0)=0$ and

$$
\lim _{t \rightarrow 0^{+}} \phi(t)=\sqrt{\sum v_{j}^{2}}>0 \text {. }
$$

By taking $\delta$ sufficiently small, $C(\delta)$ can be made less than $\frac{1}{4}$. We can therefore apply the lemmas to conclude that $\phi(t)>0$ for all $t>0$ and therefore $J(t) \neq 0$ for $t>0$. This implies that the differential of the exponential map, $d \exp _{0}$ is everywhere of maximal rank. As $g$ is complete it follows from Theorem 13.3(ii) in [6] that $\exp _{0}: T_{0} \mathbb{C}^{n} \rightarrow \mathbb{C}^{n}$ is a diffeomorphism. This completes the proof of the proposition.

Theorem 3 is an immediate consequence of the proposition and Theorem 2. 


\section{Harmonic Deformations}

In this section we consider deformations defined by deformation tensors $\Omega \in \mathcal{C}^{\infty}\left(X ; \operatorname{hom}\left(T^{0,1} X, T^{1,0} X\right)\right)$ which satisfy not only the integrability condition but also

$$
\bar{\partial}^{*} \Omega=0
$$

The formal adjoint of $\bar{\partial}$ is defined by a choice of hermitian metric on the tangent bundle of $X$.

Definition 5. A deformation tensor which satifies both (5) and (20) is called a harmonic deformation.

Harmonic deformations were introduced in the work of Kuranishi to overcome the diffeomorphism invariance of (5). In his pioneering work he showed that if $X$ is a compact, complex manifold then the space of harmonic deformations (which has a natural complex structure as an analytic subset of a complex Banach space) is the base space for the versal deformation of $\left(X, T^{0,1} X\right)$, see [10].

Motivated by Kuranishi's work in the compact case we now consider deformations of the standard complex structure on $\mathbb{C}^{n}$ which are harmonic with respect to the Euclidean metric. If $a_{i j}$ is a constant matrix then

$$
\Omega(a)=\sum_{i, j=1}^{n} a_{i j} \partial_{z_{i}} \otimes d \bar{z}_{j}
$$

is a harmonic deformation. It is not unreasonable to expect that these are the only small harmonic deformations of $\mathbb{C}^{n}$. To that end we first show that if such a deformation approaches a constant, sufficiently rapidly then it is, in fact constant. Next we consider harmonic deformations which extend smoothly to the radial compactification of $\mathbb{C}^{n}$. We denote this compactification by $\overline{\mathbb{C}^{n}}$; it is diffeomorphic to the closed unit ball in $\mathbb{C}^{n}$. A deformation has a smooth extension to $\overline{\mathbb{C}^{n}}$ if it has an asymptotic expansion at infinity of the form

$$
\Omega \sim \sum_{j=0}^{\infty} a_{j}(\sigma, \bar{\sigma}) r^{-j}
$$

with $r=|z|, \sigma=r^{-1} z$ and $a_{j} \in \mathcal{C}^{\infty}\left(\mathbb{S}^{2 n-1}\right), \quad j=0,1, \ldots$ We again conclude that all such small deformations are constant. These results suggest several interesting directions for further research which are discussed at the end of this section. 
Proposition 2. Let $\Omega$ be a harmonic deformation in $\mathbb{C}^{n}$. There exists a positive constant $\epsilon(n)$ such that if

$$
\|\Omega\|_{e} \leq \epsilon(n),
$$

and

$$
\liminf _{r \rightarrow \infty} \int_{|z|=r}|\Omega||\nabla \Omega|(1+|\nabla \Omega|) d S=0 .
$$

then $\Omega=\Omega(a)$ for an $a \in \mathrm{gl}_{n}(\mathbb{C})$.

Proof. Let $\square=\bar{\partial} \bar{\partial}^{*}+\bar{\partial}^{*} \bar{\partial}$; it follows from the hypotheses that

$$
\square \Omega=\frac{1}{2} \bar{\partial}^{*}[\Omega, \Omega]
$$

If

$$
\Omega=\sum_{i, j=1}^{n} a_{i j}(z, \bar{z}) \partial_{z_{i}} \otimes d \bar{z}_{j} \text { and } \Xi=\sum_{i, j=1}^{n} b_{i j}(z, \bar{z}) \partial_{z_{i}} \otimes d \bar{z}_{j}
$$

then we let

$$
\langle\Omega, \Xi\rangle=\sum_{i, j=1}^{n} a_{i j} \bar{b}_{i j}, \quad|\Omega|^{2}=\langle\Omega, \Omega\rangle .
$$

The chain rule implies that

$$
\square|\Omega|^{2}=\langle\square \Omega, \Omega\rangle+\langle\Omega, \square \Omega\rangle+|\nabla \Omega|^{2}
$$

Let $B_{r}$ be the ball centered at the origin of radius $r$. Integrate the left side of $(24)$ on $B_{r}$, applying Green's formula we find that

$$
\int_{B_{r}} \square|\Omega|^{2} d V=2 \Re \int_{b B_{r}}\left\langle\frac{\partial \Omega}{\partial n}, \Omega\right\rangle d S .
$$

It follows from (22) and (25) that there exists a seqeunce $r_{m} \rightarrow \infty$ such that

$$
\lim _{n \rightarrow \infty} \int_{B_{r_{m}}} \square|\Omega|^{2} d V=0 .
$$

Integrating the right side of (24) over $B_{r_{m}}$, we find that

$$
\begin{aligned}
\int_{B_{r_{m}}} \square|\Omega|^{2} d V= & \int_{B_{r_{m}}} \Re\left\langle\bar{\partial}^{*}[\Omega, \Omega], \Omega\right\rangle d V+\int_{B_{r_{m}}}|\nabla \Omega|^{2} d V \\
= & \int_{B_{r_{m}}} \Re\langle[\Omega, \Omega], \bar{\partial} \Omega\rangle d V+\int_{b B_{r_{m}}} f(\Omega, \nabla \Omega) d S \\
& +\int_{B_{r_{m}}}|\nabla \Omega|^{2} d V
\end{aligned}
$$

Here

$$
|f(\Omega, \nabla \Omega)|=O\left(|\Omega||\nabla \Omega|^{2}\right)
$$


and therefore (22) implies that this term in (27) tends to zero as $m \rightarrow$ $\infty$. Using the Cauchy-Schwarz inequality on the other term in (27) and (26) we conclude that there is a constant $C(n)$ such that

$$
\lim _{m \rightarrow \infty} \int_{B_{r_{m}}}\left[-C(n)|\Omega||\nabla \Omega|^{2}+|\nabla \Omega|^{2}\right] d V \leq 0 .
$$

If $|\Omega|<1 / C(n)$ then evidently this implies that $|\nabla \Omega|=0$ almost everywhere. As $\Omega$ is twice differentiable this implies that $\nabla \Omega \equiv 0$.

Now we consider harmonic deformations that extend smoothly to $\overline{\mathbb{C}^{n}}$.

Theorem 4. Suppose that $\Omega$ is an harmonic deformation with an asymptotic expansion at infinity as in (21). For each $0<\alpha<1$ there are positive constants, $\epsilon(n), \eta(n, \alpha)$ such that if

$$
\|\Omega\|_{e}<\epsilon \text { and }\left\|a_{0}\right\|_{C^{1, \alpha}\left(\mathbb{S}^{2 n-1}\right)}<\eta
$$

then $\Omega=\Omega(a)$ for an $a \in \mathrm{gl}_{n}(\mathbb{C})$.

Proof. By comparing terms in the asymptotic expansions, the equations (5) and (20) imply that for $k \in \mathbb{N}_{0}$ the following equations are satisfied in $\mathbb{C}^{n} \backslash\{0\}$ :

$$
\begin{aligned}
& \bar{\partial}\left(r^{-k} a_{k}\right)-\frac{1}{2} \sum_{i+j=k}\left[r^{-i} a_{i}, r^{-j} a_{j}\right]=0, \\
& \bar{\partial}^{*}\left(r^{-k} a_{k}\right)=0
\end{aligned}
$$

which imply that

$$
\square\left(r^{-k} a_{k}\right)-\frac{1}{2} \sum_{i+j=k} \bar{\partial}^{*}\left[r^{-i} a_{i}, r^{-j} a_{j}\right]=0 .
$$

The coefficients, $\left\{a_{k}\right\}$ are $n \times n$-matrix valued functions. We first consider $k=0$ : in this case (30) implies that

$$
\Delta_{S^{2 n-1}} \otimes \operatorname{Id}_{n} a_{0}-Q\left(a_{0}, \nabla_{S^{2 n-1}} a_{0}, \nabla_{S^{2 n-1}}^{2} a_{0}\right)=0
$$

on $S^{2 n-1}$. Here $Q(\alpha, \beta, \gamma)$ is a homogeneous polynomial of degree 2 . Let $G$ denote the partial inverse of $\Delta_{S^{2 n-1}}$ and $H$ the orthogonal projection onto the constant functions. Equation (31) and the fact that $H G=0$ imply that

$$
\mathcal{G}\left(a_{0}\right)=\left(\operatorname{Id}_{n}-H \otimes \operatorname{Id}_{n}\right)\left(a_{0}-G \otimes \operatorname{Id}_{n}\left(Q\left(a_{0}, \nabla_{S^{2 n-1}} a_{0}, \nabla_{S^{2 n-1}}^{2} a_{0}\right)\right)\right)=0
$$


If $0<\alpha<1$ then it is a standard result in potential theory that the map $a_{0} \rightarrow \mathcal{G}\left(a_{0}\right)$ is a smooth map from $\mathcal{C}^{1, \alpha}\left(S^{2 n-1} ; \mathrm{gl}_{n}\right)$ to itself. We define

$$
\mathcal{E}\left(a_{0}\right): \mathcal{C}^{1, \alpha}\left(S^{2 n-1} ; \mathrm{gl}_{n}\right) \longrightarrow\left(\operatorname{Id}_{n}-H \otimes \operatorname{Id}_{n}\right) \mathcal{C}^{1, \alpha}\left(S^{2 n-1} ; \mathrm{gl}_{n}\right) \oplus \mathrm{gl}_{n}
$$

setting

$$
\mathcal{E}\left(a_{0}\right)=\left(\mathcal{G}\left(a_{0}\right), H \otimes \operatorname{Id}_{n}\left(a_{0}\right)\right) .
$$

Again $\mathcal{E}$ is a smooth map and $\mathcal{E}^{\prime}(0)(a)=\left(\left(\operatorname{Id}_{n}-H \otimes \operatorname{Id}_{n}\right) a, H \otimes \operatorname{Id}_{n} a\right)$ is an isomorphism. From the inverse function theorem in a Banach space it follows that there exists an $\eta^{\prime}>0$ such that if $A \in \mathrm{gl}_{n}$ has norm less that $\eta^{\prime}$ then there is a unique $a_{0} \in \mathcal{C}^{1, \alpha}\left(S^{2 n-1} ; \mathrm{gl}_{n}\right)$ near 0 such that $\mathcal{E}\left(a_{0}\right)=(0, A)$. Of course $a_{0}=A$ solves this equation and is therefore the unique solution. This implies that there is an $\eta>0$ such that if $\left\|a_{0}\right\|_{\mathcal{C}^{1, \alpha}\left(S^{2 n-1} ; \mathrm{gl}_{n}\right)}<\eta$ satisfies (31) then $a_{0}$ is a constant matrix.

We now consider the case $k=1$, equation (30) becomes

$$
\square\left(r^{-1} a_{1}\right)-\bar{\partial}^{*}\left[a_{0}, r^{-1} a_{1}\right]=0 \text { for } r \neq 0 .
$$

If $\left|a_{0}\right|$ is sufficiently small then this is a homogeneous, constant coefficient, linear elliptic equation for $r^{-1} a_{1}$ in $\mathbb{C}^{n} \backslash\{0\}$. The function $r^{-1} a_{1}$ defines a unique homogeneous distribution of degree -1 on $\mathbb{C}^{n}$ which is a weak solution of this same equation. Since the dimension of the ambient space is at least 4 it follows from standard results on removable singularities for elliptic equations that $r^{-1} a_{1}=0$.

We can use this argument recursively to conclude that

$$
a_{j}=0 \text { for } j=1, \ldots 2 n-3 \text {. }
$$

By induction $a_{1}=\cdots=a_{j-1}=0$ for a $j<2 n-4$. By comparing coefficients in the asymptotic expansion we conclude that for $r \neq 0$ we have

$$
\square\left(r^{-j} a_{j}\right)-\bar{\partial}^{*}\left[a_{0}, r^{-j} a_{j}\right]=0 .
$$

That is $r^{-j} a_{j}$ is a homogeneous solution of order $0>-j \geq 3-2 n$ to a constant coefficient linear system which is a small perturbation of $\Delta \otimes \operatorname{Id}_{n}$. From homogeneity considerations it follows that the extension of $r^{-j} a_{j}$ to $\mathbb{C}^{n}$ as a homogeneous distribution is a weak solution of

$$
\square\left(r^{-j} a_{j}\right)-\bar{\partial}^{*}\left[a_{0}, r^{-j} a_{j}\right]=e_{j} .
$$

Where $e_{j}$ is a homogeneous distribution of degree $-j-2 \geq 1-2 n$ with support contained in $\{0\}$. As such a distribution can be represented as a differential operator applied to the $\delta$-function, the contraint on the degree implies that $e_{j}=0$. By elliptic regularity $r^{-j} a_{j}$ has a removable singularity at 0 and therefore $a_{j} \equiv 0$. 
If $a_{0}=0$ then we can apply Proposition 2 to complete the argument. If $a_{0} \neq 0$ then we need to consider the case $k=2 n-2$. This coefficient satisfies

$$
\begin{aligned}
& L_{a_{0}}\left(r^{2-2 n} a_{2 n-2}\right)=\square\left(r^{2-2 n} a_{2 n-2}\right)-\bar{\partial}^{*}\left[a_{0}, r^{2-2 n} a_{2 n-2}\right]=0, \\
& \bar{\partial}^{*}\left(r^{2-2 n} a_{2 n-2}\right)=0 .
\end{aligned}
$$

To complete the proof we show that the only solution to this system of equations is $a_{2 n-2}=0$.

The symbol of $L_{a_{0}}$ is given by

$$
\sigma\left(L_{a_{0}}\right)(\xi)=|\xi|^{2} \operatorname{Id}_{n}+q_{a_{0}}(\xi)
$$

where $q_{a_{0}}$ is an $n \times n$-matrix valued, homogeneous polynomial of degree 2 which depends linearly on $a_{0}$. A solution to the first equation in (37) has a unique extension, $A$ to $\mathbb{C}^{n}$ as a matrix valued distribution, homogeneous of degree $2-2 n$. Considerations of homogeneity show that this distribution satisfies

$$
L_{a_{0}} A=C_{0} \otimes \delta_{0}
$$

where $C_{0}$ is a constant matrix. The Fourier transform of $A$ is a matrix valued distribution, $\hat{A}(\xi)$ which is homogeneous of degree -2 . Since the dimension of the ambient space is at least 4 this distribution is simply integration with respect to a function in $\mathcal{C}^{\infty}\left(\mathbb{C}^{n} \backslash\{0\} ; \mathrm{gl}_{n}\right)$, homogeneous of degree -2 . The Fourier transform of (39) gives

$$
\sigma\left(L_{a_{0}}\right)(\xi) \hat{A}(\xi)=C_{0} .
$$

In other words,

$$
\hat{A}(\xi)=\left[\sigma\left(L_{a_{0}}\right)(\xi)\right]^{-1} C_{0} .
$$

If $E_{a_{0}}$ denotes the inverse transform of $\left[\sigma\left(L_{a_{0}}\right)(\xi)\right]^{-1}$, the unique fundamental solution for $L_{a_{0}}$ which is homogeneous of degree $2-2 n$ then this implies that

$$
A(z, \bar{z})=E_{a_{0}}(z, \bar{z}) C_{0} .
$$

It is a simple consequence of classical results in the theory of distributions that the map

$$
\left.a_{0} \longrightarrow E_{a_{0}}\right|_{S^{2 n-1}}
$$

is a smooth map from $\mathrm{gl}_{n}$ to $\mathcal{C}^{\infty}\left(S^{2 n-1}\right)$, see [7] which implies that

$$
E_{a}(z, \bar{z})=c_{n} \operatorname{Id}_{n}|z|^{2-2 n}+e_{a_{0}}(z, \bar{z})
$$

where all derivatives of $\left.e_{a_{0}}\right|_{S^{2 n-1}}$ are bounded by $\left\|a_{0}\right\|_{e}$. Using the second equation in (37) it now follows that, if $\left\|a_{0}\right\|_{e}$ is sufficiently small, then $v C_{0}=0$ for all $v \in \mathbb{C}^{n}$ which implies that $a_{2 n-2}=0$ as well. 
The first non-constant term in the asymptotic expansion of $\Omega$ is therefore $r^{1-2 n} a_{2 n-1}$ which again allows the application of Proposition 2 to complete the proof of the theorem.

Remark 2. In the proof we do not use the existence of a complete asymptotic expansion: only the terms of degree greater than $1-2 n$ with an appropriate error estimate are needed. In the context of deformations of complex structures it is natural to assume that the solution is bounded at infinity. It is not necessary to restrict the asymptotic expansion at infinity to be in terms of integral powers of $r^{-1}$. Suppose that a solution has an expansion with finitely polyhomogeneous terms of degrees greater than $2-2 n$ and less than 0 . Starting with the term of highest degree we could use the removable singularities argument, applied above to show recursively that all terms of degree greater than $2-2 n$ vanish. Terms of degree less than $2-2 n$ are handled as before by using Proposition 2.

Remark 3. It seems likely that the smallness hypothesis

$$
\left\|a_{0}\right\|_{C^{1, \alpha}(\mathbb{S} 2 n-1)}<\eta
$$

can be weakened to $\left\|a_{0}\right\|_{C^{\alpha}\left(\mathbb{S}^{2 n-1}\right)}<\eta$.

Remark 4. A bounded harmonic function in $|z|>r$ automatically has an asymptotic expansion like that posited in Theorem 4 . It seems quite an interesting question whether or not the analogous result is true for harmonic deformation tensors.

Remark 5. Using the changes of coordinate $w=z|z|^{\gamma}$ to push forward the complex structure of $\mathbb{C}^{n}$ it is easy to construct deformation tensors satisfying the integrability equation which nonetheless have isolated singular points. This shows that the structure of singularities for the $\bar{\partial}$-equation does not persist under the non-linear perturbation giving rise to the integrability condition. Is there a removable singularities theorem for harmonic deformation tensors similar to that for harmonic functions?

Remark 6. Do harmonic deformation tensors with a smooth extension to $\overline{\mathbb{C}^{n}}$ define a slice for the action of Diff $\left(\overline{\mathbb{C}^{n}}\right)$ on the set of integrable deformations with a smooth extension to $\overline{\mathbb{C}^{n}}$ ? More geometrically, does Theorem (4) imply that $\mathbb{C}^{n}$ is rigid among small deformations of $\mathbb{C}^{n}$ which extend smoothly to $\overline{\mathbb{C}^{n}}$ ?

\section{ACKNOWLEDGEMENT}

Research of both authors partially supported by NSF grant DMS9623040 . 


\section{REFERENCES}

[1] L. Ahlfors and L. Bers, Riemann's mapping theorem for variable metrics, Annals of Math.(2) 72 (1960), 385-404.

[2] Frederick P. Gardiner, Teichmüller theory and quadratic differentials, John Wiley, New York, NY, 1987.

[3] H. Grauert, On Levi's problem and the imbedding of real analytic manifolds, Annals of Math.(2) 68 (1958), 460-472.

[4] Richard Hamilton, Deformations of complex structures on manifolds with boundary. I: The stable case, JDG 12 (1977), 1-45.

[5] Reese Harvey, Holomorphic chains and their boundaries, Proceeding of Colloquia in Pure Math. XXX (1977), 309-382.

[6] S. Helgason, Differential geometry, lie groups and symmetric spaces, Academic Press, New York, 1978.

[7] L. Hörmander, The analysis of linear partial differential operators, vol. 3, Springer-Verlag, Berlin, Heidelberg, New York, Tokyo, 1985.

[8] Garo K. Kiremidjian, Deformations of complex structures on certain noncompact complex manifolds, Annals of Math.(2) 98 (1973), 411-426.

[9] Kunihiko Kodaira, Complex manifolds and deformation of complex structures, Springer Verlag, Berlin, 1986.

[10] M. Kuranishi, Deformations of compact complex manifolds, séminaire de mathématiques supérieures, no. 39, Les Presses de l'Université de Montréal, Montréal, 1971.

[11] Y. T. Siu N. M. Mok and S. T. Yau, The Poincaré Lelong equation on complete Kähler manifolds, Compositio Math. 44 (1981), 183-218.

[12] Yong Ouyang, Deformation of Stein manifolds, PhD thesis, Univ. of Penn. (1999), 1-54.

Department of Mathematics, University of Pennsylvania

E-mail address: cle@math.upenn.edu

Department of Mathematics, University of Pennsylvania

E-mail address: ouyang@math.upenn.edu 